\title{
Incubation strategies of the Black-necked Crane (Grus nigricollis) in relation to ambient temperature and time of day
}

Lixun Zhang ${ }^{1,2^{*}}$, Bei $\mathrm{An}^{3}$, Meilin Shu' ${ }^{1}$, Changming Zhao ${ }^{1}$, Xiaojun Yang ${ }^{4^{*}}$, Yila Suo ${ }^{5}$, Yongjun Se ${ }^{5}$ and Xilite Dabu ${ }^{5}$

\begin{abstract}
Background: The behavior of cranes reflects many of their survival strategies, but little has been known of the incubation strategies of cranes, in which both parents share incubation duties, in response to cold temperatures in alpine environments. The lack of information may restrict the effective conservation of the threatened Black-necked Crane (Grus nigricollis), a biparental bird nesting in high elevation wetlands.
\end{abstract}

Methods: We directly observed and used infrared video cameras from 2014 to 2015 to study the incubation behavior and quantitatively measured the frequency and details of egg turning behavior in the Black-necked Crane at the Yanchiwan National Nature Reserve in western Gansu Province, China.

Results: At lower ambient temperatures in the morning, crane parents spent more time on the nest with less recess frequency and prolonged on-bout duration, while at higher temperatures around noon, the parents had more frequent recesses from incubation and shorter periods between nest exchanges. They adjusted the amount of time incubating by varying the recess frequency and the length of on-bout duration. Mean nest attendance and egg turning frequency of females were significantly higher than those of the males. The nest attendance and on-bout duration of females showed a significantly negative relationship with those of males. The two parents responded differently to the change of temperature. Females spent more time on the nest at lower morning temperatures, while males increased their time on the nest at higher temperatures after noon. Higher incubation recess frequency and egg turning frequency were observed at noon, probably because parents spent more time foraging, taking advantage of the lower egg cooling rate.

Conclusion: Both Black-necked Crane parents in the alpine environment adjusted their behavior in response to the thermal requirements of eggs and the weather conditions experienced. Our findings demonstrate that parents of this species incubated in different but complementary ways and efficiently enhanced egg care in a dynamic environment, so as to maximize benefits from the warm portion of the day and the intense solar radiation while minimizing the cost of rewarming eggs and the risks of cooling eggs.

Keywords: Time of day, Ambient temperature, Egg-turning frequency, On-bout duration, Nest attendance, Recess frequency, Biparental bird

\footnotetext{
*Correspondence: zhanglixun@|zu.edu.cn; yangxj@mail.kiz.ac.cn

1 School of Life Sciences, Lanzhou University, Lanzhou 730000, China

${ }^{4}$ State Key Laboratory of Genetic Resources and Evolution, Kunming

Institute of Zoology, Chinese Academy of Sciences, Kunming 650233,

China

Full list of author information is available at the end of the article
} provided you give appropriate credit to the original author(s) and the source, provide a link to the Creative Commons license, and indicate if changes were made. The Creative Commons Public Domain Dedication waiver (http://creativecommons.org/ publicdomain/zero/1.0/) applies to the data made available in this article, unless otherwise stated. 


\section{Background}

The majority of extant avian species must defend and incubate eggs until hatching (Weathers and Zaun 2010). Incubation may provide various evolutionary benefits for birds, including a decreased incubation period, accelerated rate of embryonic development, and greater management of incubation conditions (Deeming 2002). Incubation is energetically costly for birds because incubating parents must balance the thermal needs of the eggs with self-maintenance (Smith et al. 2012). Ambient temperature affects energetic costs during incubation and embryonic development during recesses from incubation (Conway and Martin 2000). However, incubation behavior is often neglected as a method for investigating relationships between animals and their environment for conservation and many of their survival strategies and adaptations (Spiegel et al. 2012). Most studies of incubation rhythms in birds have been conducted in moderate environments (Camfield and Martin 2009) and studies under alpine environments have focused mainly on uniparental incubators. However, the situation in species with biparental incubation is more complex due to the potentially different responses of each parent to changes in the cost of incubation (Smith et al. 2012). Spencer et al. (2010) emphasized that birds may exhibit sexual differences regarding incubation effort in relation to variation in their own developmental processes and current environmental conditions. Very few studies, however, have investigated the ambient temperature influence on the relative incubation contribution by each sex (Conway and Martin 2000; Bulla et al. 2014), especially in alpine environments.

Egg turning is unique to birds and crucial for embryonic development in most avian species (Shaffer et al. 2014). Optimum egg turning frequency during incubation has been of interest since the observations of feral fowl incubation behavior by Elibol and Brake (2006). Regulation of egg temperature through egg turning by incubating parents is crucial to both the survival and proper development of avian embryos. However, egg turning behavior has not been studied as widely as parental attendance pattern, egg physiology, or hatching success (Shaffer et al. 2014). In addition, egg turning frequency in wild birds is rarely studied due to difficulty of observations in darkness (Weathers and Zaun 2010).

We investigated these issues in a population of the Black-necked Crane (Grus nigricollis), a species breeding exclusively on the high-altitude wetlands of the Qinghai-Tibetan Plateau (Li and Bishop 1999). The species is a monogamous and long-lived migrant waterbird, with pair bonds lasting for years. Both males and females participate in nest building, incubation, and rearing nestlings and fledglings. Early observations suggested that males and females contribute almost equally to incubation with regard to time spent on the nest (Zhang et al. 2017a). Several studies have been attempted to determine the incubation rhythms of the Black-necked Crane with direct observations and data analysis (Kuang et al. 2007; Yang et al. 2007). However, few data are available on the incubation strategies of biparentally incubating cranes in response to cold temperatures in alpine environments. Such strategies could relate to nest attendance, recess frequency, on-bout duration and egg turning frequency. Those behaviors could alter egg temperatures and the time that developing embryos are exposed to cold temperatures. In the present study, we investigated the effects of time of day and variation in ambient temperature on male and female incubation behaviors in a cold environment at the Yanchiwan National Nature Reserve, western Gansu Province, China.

\section{Methods}

\section{Study species and study site}

The Black-necked Crane is a large ground-nesting bird that breeds only on high-altitude wetlands from Ladakh north to Gansu (Bishop et al. 2012). Their average clutch size is two eggs, with one egg laid per day, and the incubation period ranges from 30 to 34 days (Zhang et al. 2017a). For details about the study area see Zhang et al. (2017b).

\section{General field methods}

We located 20 nests (10 nests in 2014, 10 nests in 2015) at the Yanchiwan National Nature Reserve. Presence or absence at the nest was detected by observation and recorded using remote infrared thermistor sensor systems (FC-5210 mm, MMS Trail Camera, Shenzhen Baird Share Co., Ltd). Each sensor system consisted of a small $(9.4 \mathrm{~cm} \times 14.6 \mathrm{~cm})$ weatherproof miniature video camera with twelve R6 AA size batteries and a 32 G memory card allowing a continuous recording of 4-5 days. These cameras were programmed on a motion sensor with $1280 \times 720$ video size, high sensitivity, 10 s triggering interval, $60 \mathrm{~s}$ video length and $24 \mathrm{~h}$ operation mode. Cameras were placed 2-3 $\mathrm{m}$ away from nests, and were elevated $1.5-2 \mathrm{~m}$ above the ground with a metal stake. The data were also confirmed by direct observation. The ambient temperature was measured by a thermo-probe, which was placed in areas with similar protection from the wind and aspect as the nest and around $25 \mathrm{~cm}$ away from the nest scrape at ground level. The probe was connected to a data logger (Tinytag, Gemini Data Loggers Ltd.). Temperatures were logged at $1 \mathrm{~min}$ intervals with an accuracy of $0.1{ }^{\circ} \mathrm{C}$. Each video-monitored nest was visited every four days to change batteries and memory cards and download temperature data when both parents 
left their nest for foraging or other activities. Cameras were collected after each recording session and videotapes were later reviewed for scoring. From these videos, we were able to determine the time of initiation and calculate each recess and on-bout duration. Sex identification referred to the method described by Kuang et al. (2007) and Zhang et al. (2017a). For each of seven periods during the day $(6: 30-8: 29,8: 30-10: 29,10: 30-12: 29$, $12: 30-14: 29, \quad 14: 30-16: 29, \quad 16: 30-18: 29$ and 18:3020:29 h, Beijing time), we calculated: (1) nest attendance, estimated as the percentage of total time when the eggs were incubated by each parent (individual nest attendance) and pair (total nest attendance), (2) recess frequency, estimated as the average number of breaks taken by each parent (individual recess frequency) and pair (total recess frequency), (3) on-bout duration, estimated as the mean length (in minutes) of uninterrupted incubation by either parent (individual on-bout duration) and pair (total on-bout duration), and (4) egg turning frequency, estimated as number of egg turning times by each parent (individual egg turning frequency) and pair (total egg turning frequency). The behavior was considered as two behaviors if one behavior occurred during two time periods. The average ground temperature outside the nests was taken as the ambient temperature for each period.

\section{Statistical analysis}

To determine whether four incubation behaviors and ambient temperatures varied with time, we examined relationships of nest attendance, recess frequency, onbout duration and egg turning frequency with time of day using curvilinear regression. Ambient temperature, nest attendance, recess frequency, on-bout duration and egg turning frequency failed to meet assumptions for a parametric test. We also included year (2014 and 2015) as a fixed effect. Thus, yearly difference of ambient temperature was tested by Mann-Whitney $U$ test, and sexual differences of nest attendance, recess frequency, on-bout duration and egg turning frequency were tested by nonparametric paired tests. Means are reported with standard error, and all significance values are at 0.05 level based on two-tailed tests. All the statistical analyses were performed by the software SPSS (version 22.0, IBM 2013).

To explore the influences of potential factors on incubation behaviors (nest attendance, recess frequency, on-bout duration, and egg turning frequency), we used generalized linear mixed models (GLMMs) in SPSS. To control for repeated measures of incubation behaviors at a given nest, we included nest identity as a random effect. Year (2014 and 2015), date, days of incubation and time of day were also added as fixed effects since they might potentially influence the incubation behavior (Camfield and Martin 2009). Partner's behavior was also a fixed factor, and partner's behavior $\times$ time period interaction was included to determine if the relationship between the incubating crane's behaviors on the nest and partner's behaviors varied over time. Square temperature was added into the model as a fixed effect because there was a significant quadratic relationship between mean ambient temperature and time of day $\left(R^{2}=0.31, F_{2,664}=149.57\right.$, $p<0.001)$. No linear correlations were found between ambient temperature and incubation behaviors (Additional File 1: Fig. S1). Thus, no interaction of ambient temperature $\times$ temperature zone was added into the models. Akaike's information criteria (AIC-based model selection) were used for model selection.

\section{Results}

A total of 153 incubation days (1334 h with 667 time periods) for Black-necked Cranes were monitored in 11 nests ( 5 nests in 2014 and 6 nests in 2015) between 20 April 2014 and 15 June 2015. The mean number of days for each nest was $13.91 \pm 2.15$ (range 5-25 days) and mean number of periods was $121.27 \pm 23.39$ (range 12-134 periods). Throughout the breeding season, the mean daytime ambient temperature was $10.13 \pm 0.27{ }^{\circ} \mathrm{C}$ with the highest temperature $30.24{ }^{\circ} \mathrm{C}$ and the lowest $-1.63{ }^{\circ} \mathrm{C}$, occasionally varying by more than $30{ }^{\circ} \mathrm{C}$ in one day. There were no marked differences in mean ambient temperature between years (2014: $10.15 \pm 0.34{ }^{\circ} \mathrm{C}$; 2015: $10.10 \pm 0.44{ }^{\circ} \mathrm{C}$; Mann-Whitney $\mathrm{UZ}=-0.60$, $p=0.547)$. The highest mean daytime temperature occurred between 14:30 and 16:29 h $\left(15.01 \pm 0.61{ }^{\circ} \mathrm{C}\right.$, Fig. 1) and the lowest occurred between 06:30 and 08:29 h $\left(2.49 \pm 0.52{ }^{\circ} \mathrm{C}\right.$, Fig. 1). Moreover, the mean ambient temperature was significantly different among different time periods (Kruskal-Wallis $X=242.03, p<0.001$ ).

\section{Nest attendance}

For every 2-h interval, the mean total nest attendance was $90.72 \pm 0.57 \%$ and was significantly quadratic to time of day $\left(R^{2}=0.03, F_{2,664}=9.46, p<0.001\right.$; Fig. 1$)$. The highest nest attendance was $95.46 \pm 1.15 \%$ between $06: 30$ and 08:29 $\mathrm{h}$ while the lowest was $86.71 \pm 1.64 \%$ between $10: 30$ and 12:29 $\mathrm{h}$. The mean female nest attendance was significantly higher than that of males (females: $47.88 \pm 1.32 \%$; males: $42.85 \pm 1.32 \%$; Wilcoxon $Z=-2.01, p=0.044$ ). Among the seven time periods, the highest female nest attendance $(58.88 \pm 4.28 \%)$ occurred between 06:30 and $08: 29 \mathrm{~h}$, and the lowest $(40.64 \pm 3.19 \%)$ between 12:30 and 14:29 h (Fig. 1). Conversely, the highest male nest attendance $(47.69 \pm 3.32 \%)$ was between $12: 30$ and $14: 29 \mathrm{~h}$, and the lowest $(36.60 \pm 4.15 \%)$ was between 06:30 and 08:29 h (Fig. 1). Meanwhile, we found a 


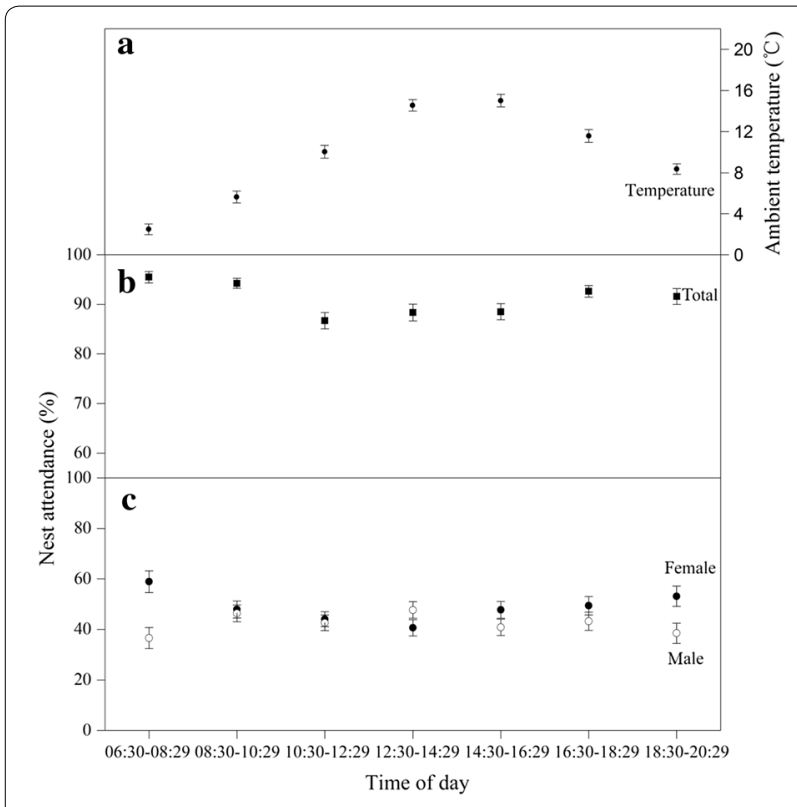

Fig. 1 Changes of ambient temperature (mean $\pm \mathrm{SE}$; $\mathbf{a}$ ), and total nest attendance (mean \pm SE) of two parents $(\mathbf{b})$, females (filled circles; c), and males (open circles; $\mathbf{c}$ ) with time of day. The number of nests used in the analyses is 11 except during 18:30-20:29 when it is 10, also applying to Figs. 2, 3, and 4

quadratic relationship between female nest attendance and time of day $\left(R^{2}=0.02, F_{2,664}=6.46, p=0.002\right)$.

Both female and male optimal nest attendance models included date and square temperature (Additional File 2: Tables S1 and S2). Both female and male nest attendances were significantly positively related to year, date and time of day and negatively to days of incubation, but not related to temperature or square temperature (Tables 1 , 2 ). A significant negative relationship was found between female and male nest attendance $(\beta=-0.63 \pm 0.05$, $p<0.001$, Table $1 ; \beta=-0.56 \pm 0.04, p<0.001$, Table 2).

\section{Recess frequency}

The total female and male recess frequency had a significant quadratic relation to time of day $\left(R^{2}=0.02\right.$, $\left.F_{2,664}=6.59, p=0.001\right)$. Among the seven time periods, the lowest total recess frequency $2.57 \pm 0.14$ times was between 06:30 and 08:29 $\mathrm{h}$ while the highest was $3.26 \pm 0.12$ times between 10:30 and 12:29 h. Both parents took recesses more frequently near noon. There were no significant differences between mean female and male recess frequency $(1.50 \pm 0.04$ vs. $1.39 \pm 0.04$ times, Wilcoxon $Z=-1.69, p=0.092)$. The highest female recess frequency was $1.72 \pm 0.10$ times between $10: 30$ and $12: 29 \mathrm{~h}$ while the lowest was $1.38 \pm 0.10$ times between 18:30 and 20:29 h (Fig. 2). The mean male recess frequency had a significant quadratic relationship to time of day $\left(R^{2}=0.01, F_{2,664}=3.85, p=0.022\right)$. The highest male recess frequency was $1.53 \pm 0.10$ times between 10:30 and 12:29 while the lowest was $1.16 \pm 0.10$ times between 06:30 and 08:29 h (Fig. 2).

Both optimal female and male recess frequency models included date and square temperature (Additional File 2: Tables S1 and S2). Female recess frequency was significantly positively related to year, date, time of day, and negatively related to day of incubation, temperature, square temperature, male recess frequency and male by time (Table 1). Male recess frequency was significantly positively related to year, date, and time of day, and negatively related to day of incubation, temperature, square temperature and female by time (Table 2).

\section{On-bout duration}

The longest on-bout duration was $57.90 \pm 4.17 \mathrm{~min} /$ bout between $06: 30$ and 08:29 h while the shortest was $37.96 \pm 2.09 \mathrm{~min} /$ bout between $10: 30$ and 12:29 h (Fig. 3). Mean total on-bout duration had a significantly quadratic relation with time of day $\left(R^{2}=0.02\right.$, $\left.F_{2,664}=6.82, p=0.001\right)$. There were no significant differences in mean daily on-bout duration between females and males (females: $40.93 \pm 1.44 \mathrm{~min} /$ bout; males: $37.66 \pm 1.38 \mathrm{~min} /$ bout, $n=930$ bouts from 11 nests, Wilcoxon $Z=-1.17, p=0.243)$. The longest female onbout duration was $51.99 \pm 4.99 \mathrm{~min} /$ bout between 06:30 and $08: 29 \mathrm{~h}$ while the shortest was $31.39 \pm 2.97 \mathrm{~min} /$ bout between 12:30 and 14:29 h (Fig. 3). Female on-bout duration had a significantly quadratic relation with time of day $\left(R^{2}=0.02, F_{2,664}=7.03, p=0.001\right)$. The longest male onbout duration was $42.41 \pm 3.52 \mathrm{~min} /$ bout between 12:30 and 14:29 $\mathrm{h}$ while the shortest was $33.96 \pm 3.07 \mathrm{~min} /$ bout between 10:30 and 12:29 h (Fig. 4).

Both optimal female and male on-bout duration models included date and square temperature (Additional File 2: Tables S1 and S2). Both female and male on-bout duration were significantly positively related to year, date and time of day (Tables 1,2). Female on-bout duration was significantly negatively related to day of incubation and male on-bout duration but not to temperature, square temperature or male by time, while male onbout duration was significantly negatively related to day of incubation, female on-bout duration and female by time but not to temperature or square temperature (Tables 1, 2). Female on-bout duration and male onbout duration showed a significant negative relationship $(\beta=-0.55 \pm 0.07, p<0.001$, Table $1 ; \beta=-0.45 \pm 0.06$, $p<0.001$, Table 2).

\section{Egg turning frequency}

A crane turns its eggs by standing up, moving backward to the nest's rim, reaching down and hooking the farthest egg with its bill and feet, then drawing the egg backward, 


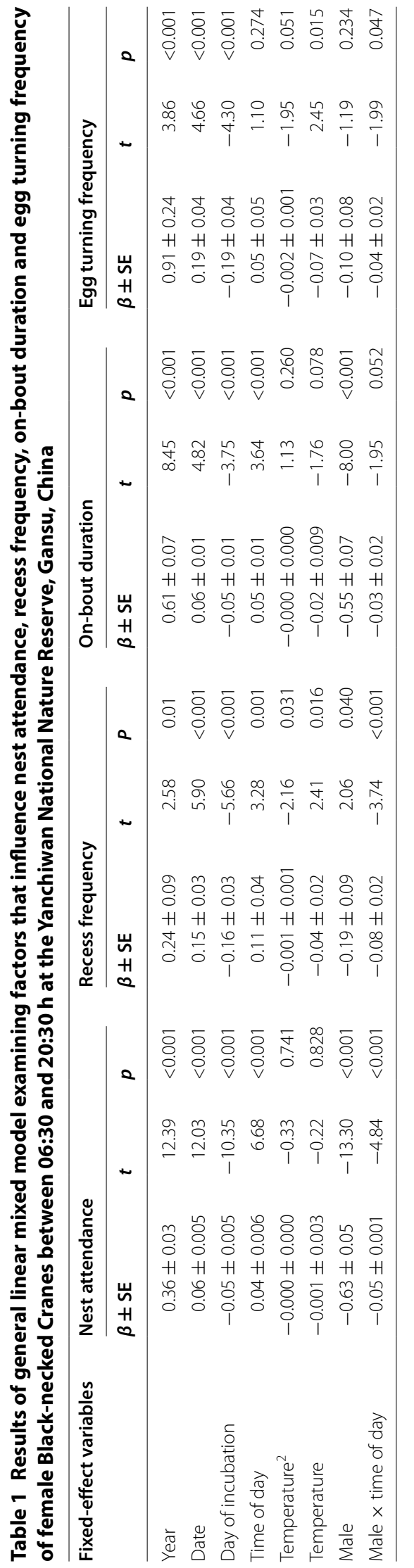




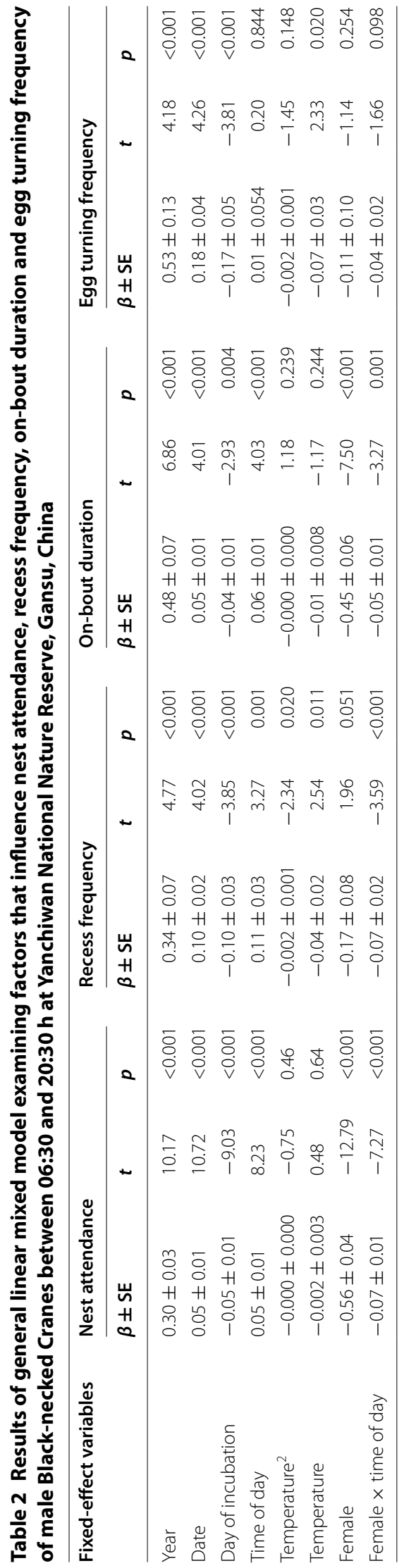




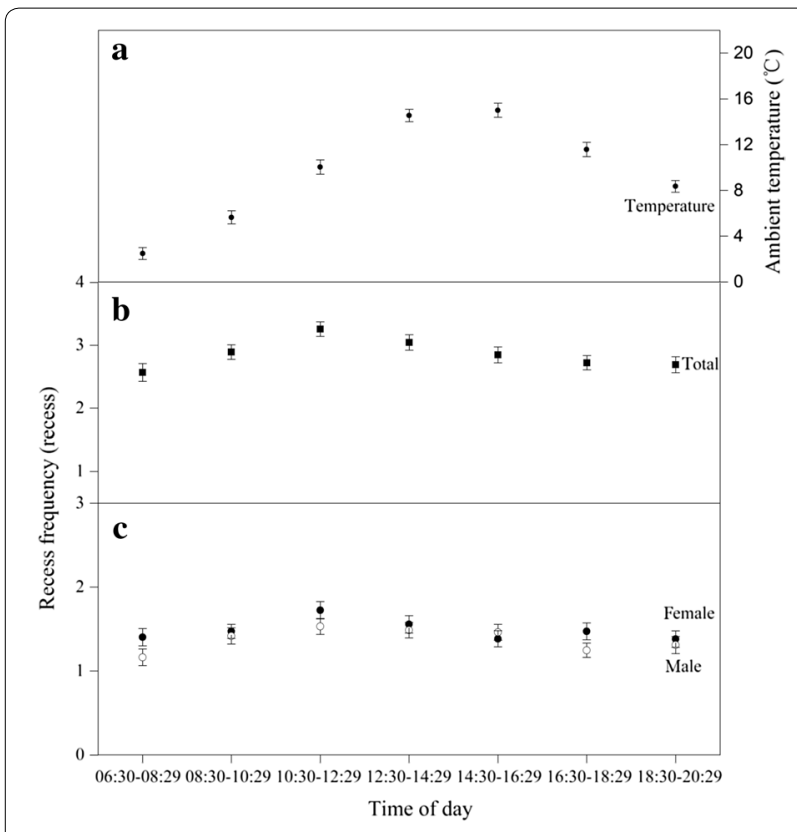

Fig. 2 Changes of ambient temperature (mean $\pm S E ;$ a), and total recess frequency (mean $\pm \mathrm{SE}$ ) of two parents $(\mathbf{b})$, females (filled circles; c), and males (open circles; $\mathbf{c}$ ) with time of day

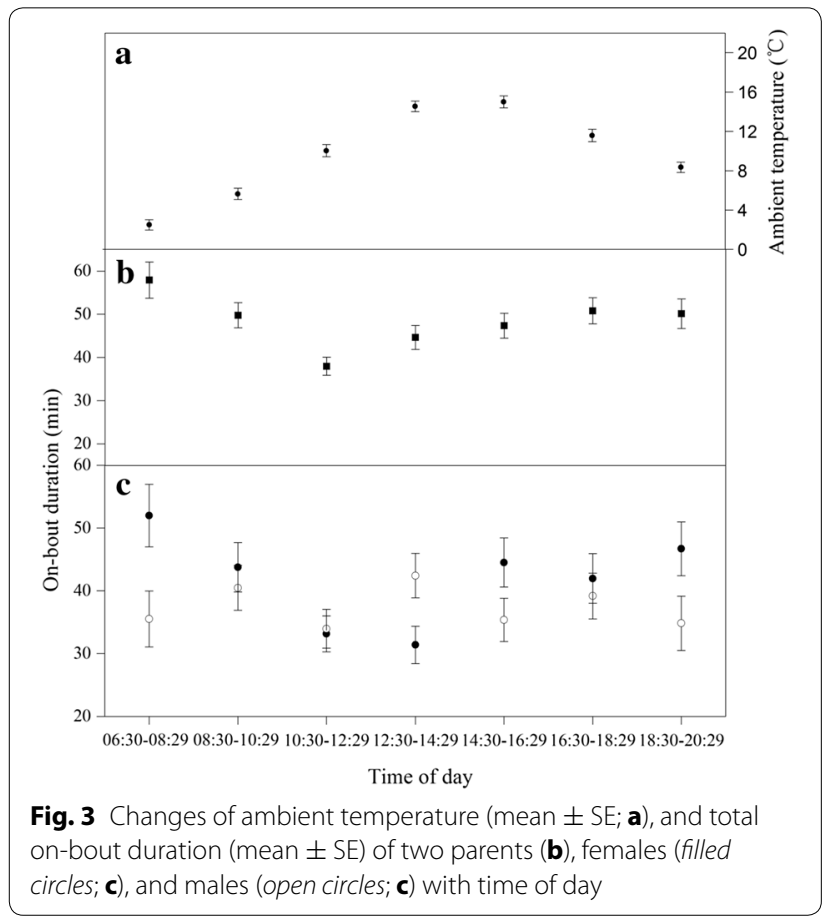

thereby rolling it (Additional File 3: Video 1). Total egg turning frequency was $3.75 \pm 0.08$ turns every 2-h interval and had a significantly quadratic relation with time of day $\left(R^{2}=0.04, F_{2,664}=12.20, p<0.001\right.$; Fig. 4). The lowest egg turning frequency ( $3.07 \pm 0.19$ turns) was between 18:30 and 20:29 $\mathrm{h}$ and the highest $(4.50 \pm 0.22$ turns) was between 12:30 and 14:29 h. Mean female egg turning frequency was significantly greater than mean male egg turning frequency (females: $2.01 \pm 0.07$ turns; males: $1.74 \pm 0.07$ turns, Wilcoxon $Z=-2.44, p=0.015$ ). Both mean female and male egg turning frequencies had a significantly quadratic relation with time of day (female: $R^{2}=0.01, F_{2,664}=3.94, p=0.020$; male: $R^{2}=0.02$, $F_{2,664}=5.30, p=0.005$; Fig. 4). Both the highest female and highest male egg turning frequencies occurred between 12:30 and 14:29 h (females: $2.29 \pm 0.19$ turns; males: $2.20 \pm 0.18$ turns), and the lowest female and male egg turning frequencies were between 18:30 and 20:29 h (females: $1.70 \pm 0.17$ turns; males: $1.37 \pm 0.20$ turns).

Both optimal female and male egg turning frequency models included date and square temperature (Additional File 2: Tables S1 and S2). Both female and male egg turning frequencies were significantly related to year, date, and were negatively related to day of incubation and temperature. Female and male egg turning frequencies showed no significant negative relationship $(\beta=-0.10 \pm 0.08, p=0.234$, Table $1 ; \beta=-0.11 \pm 0.10$, $p=0.254$, Table 2).

\section{Discussion}

In the alpine environment, an optimal incubation schedule trades off the incubators' energetic cost against the demand for eggs to be maintained suitably warm. This complicated trade-off can be driven by various influences such as incubation energetic needs and the egg chilling tolerance. Our study demonstrated a multimodal pattern in the parental incubation behaviors of the Black-necked Crane in response to cold temperatures in an alpine environment throughout the entire incubation period. The highest nest attendance, the longest on-bout duration and the lowest total recess frequency all occurred in the early morning (06:30-08:29 h) when the mean ambient temperature is the lowest. This is consistent with parent inactivity of the Black-necked Crane in the morning during incubation in northern Tibet (Kuang et al. 2007). The highest total recess frequency, lowest nest attendance and the shortest on-bout duration occurred between 10:30 and 12:29 $\mathrm{h}$. Meanwhile, the highest egg turning frequency of both parents occurred between 12:30 and $14: 29 \mathrm{~h}$ when the mean ambient temperature was high. We suggest that biparental behaviors of the Black-necked Crane may reflect the predictable patterns in temperature and solar radiation throughout the alpine day. Both parents took the longest and highest recess frequencies in the warmer hours of the day, and nests were incubated more constantly during the cooling temperatures. Similar parental care was reported in the female King Eider 


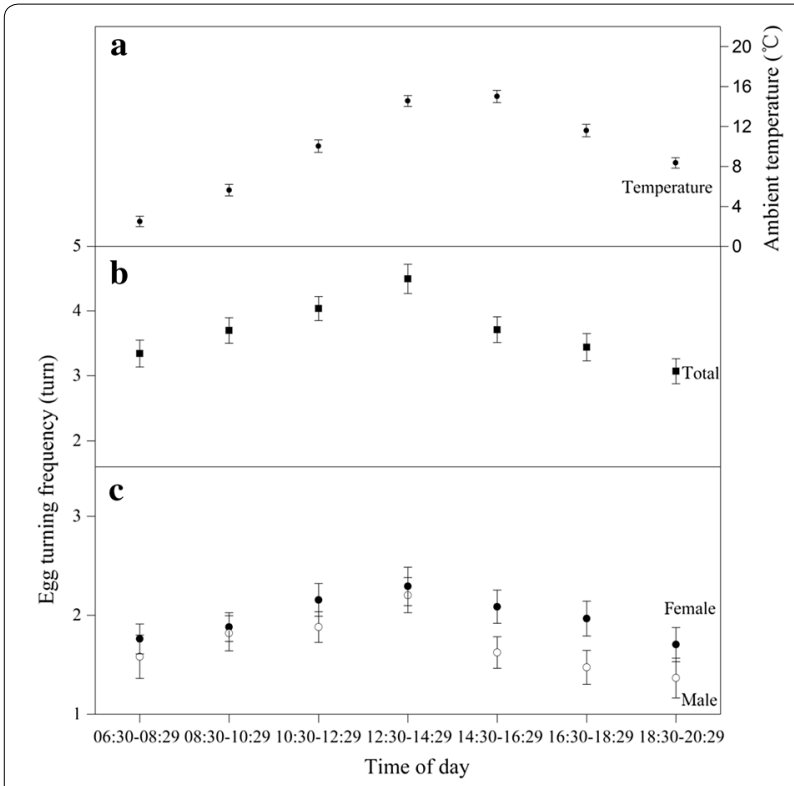

Fig. 4 Changes of ambient temperature (mean $\pm S E ; \mathbf{a}$ ), and total egg turning frequency (mean \pm SE) of two parents (b) females (filled circles; c), and males (open circles; $\mathbf{c}$ )

(Somateria spectabilis) which preferred to take advantage of the warmest part of the day to reduce cooling of eggs, despite that their predators were also more active during the same period (Bentzen et al. 2010). We also noticed that Black-necked Cranes were more active between 10:30 and 12:29 h, much earlier than the most active periods of this species in northern Tibet (Kuang et al. 2007). This difference might be explained as incubation behavior being functionally driven by local foraging conditions (Bentzen et al. 2010).

Total hourly egg turning frequency was 1.87 turns, which fell in hourly egg turning rates of three species, e.g. Cassin's Auklet (Ptychoramphus aleuticus), Western Gull (Larus occidentalis), and Laysan Albatross (Phoebastria immutabilis) (Shaffer et al. 2014). The highest egg turning frequencies of both females and males occurred between 12:30 and 14:29 $\mathrm{h}$, and the lowest frequencies of both parents were between 18:30 and 20:29 h, suggesting that ambient temperature plays a significant role in egg turning behavior for Black-necked Cranes. Both crane parents took advantage of the warmest session of the day when solar radiation was the most intense. This timing indicated they were sensitive to temperature variation within days and modified their behavior so as to maximize benefits from the most intense solar radiation by taking incubation recesses during the warmest part of the day for saving energy. Such behavior appears to be adaptive since at higher ambient temperatures, rate of egg cooling was slower, permitting parents to stay off the nest for a longer time (Deeming 2002).
Female and male nest attendance, on-bout duration, and egg turning frequency showed a significantly negative relationship (Tables 1,2). We also found that on-bout durations were generally shorter and nest attendance was lower for males than for females during the day. This result was consistent with reports that females conducted a greater portion of incubation than males (Kuang et al. 2007). The highest female nest attendance was from $06: 30$ to $08: 29 \mathrm{~h}$, when females may be short of energy after an overnight incubation. This finding is similar to the incubation behavior during early morning for females of a uniparental species Horned Lark (Eremophila alpestris) breeding in alpine tundr (Camfield and Martin 2009), but was in contrast to the uniparental incubation pattern for the female King Eider, which took more recesses at low temperatures because recesses were driven by metabolic expenses and local foraging conditions (Bentzen et al. 2010). Our study demonstrated that female Black-necked Cranes shifted priority between self-maintenance and incubation demands depending on various temperature conditions and their parental cooperation. Similarly, Magrath et al. (2005) reported that females were more sensitive to the thermal status and demands of the clutch than males.

Female cranes tended to reduce attendance during the warmest period of day, consistent with the biparental incubation pattern of Arctic-breeding female Semipalmated Sandpipers (Calidris pusilla), which were more often off nest during the warmer period of the day when foraging might be easier (Bulla et al. 2014). However, the increase in nest attendance by males may represent compensation for the reduced attentiveness by the female, consistent with results of Magrath et al. (2005). The optimal contribution by one parent should depend on the level of investment by their partner, with a reduction in effort by one parent predicted to result in partial compensation by the other. Sexual differences in the level of resting and movement observed in our study can be explained by the dominant role of females in incubation. Bulla et al. (2014) also suggested that the female-biased extent of incubation might be compensated for a more favorable timing of incubation. Biparental care and complementary sexual roles counter the high risk of predation, with males assuming more vigilance and nest defense behaviors, and females spending more time on eggs (Kuang et al. 2007). The strong attachment of the parents to their nest helps to ensure the egg is well protected. A shortcoming of our study was that the behavior of birds was not monitored when birds were absent from the nest or during night. More complex interactions may also be involved in biparental incubation behaviors. 


\section{Conclusions}

Black-necked Cranes are sensitive to temperature variations within the day and can adjust their behavior so as to maximize benefits from the warmest session of the day and the most intense solar radiation, with decreased cooling of eggs. Our findings demonstrate that Blacknecked Crane parents incubated in different but complementary ways, and efficiently enhanced egg care in a dynamic environment. Their incubation behaviors were modified in response to the thermal requirements of eggs and the weather conditions experienced.

\section{Additional files}

Additional file 1: Fig S1. Mean \pm SE of nest attendance (a), recess frequency (b), on-bout duration (c) and egg turning frequency (d) varied with ambient temperature for Black-necked Cranes (females: line with filled circles; males: line with open circles) in Yanchiwan National Nature Reserve, Gansu, China, in 2014 and 2015.

Additional file 2: Table S1. Summary of model selection results for incubation rhythms for female Black-necked Cranes in Yanchiwan national nature reserve in 2014 and 2015. All models include year, day of incubation, temperature, partner's behavior and partner's behavior $x$ time of dayas fixed effects. Nest identity was included as a random factor in all models. Table S2. Summary of model selection results for incubation rhythms for male Black-necked Cranes in Yanchiwan national nature reserve in 2014 and 2015. All models include year, day of incubation, temperature, partner's behavior and partner's behavior $x$ time of day as fixed effects. Nest identity was included as a random factor in all models.

Additional file 3. A crane is turning its egg.

\section{Authors' contributions}

$X Y$ and $L Z$ conceived, designed and directed the experiments. BA and LZ coordinated this study, and wrote and revised the manuscript. MS performed the statistical analyses, YS, YS and XD helped with nest site location and infrared cameras set, and revised the manuscript. All authors read and approved the final manuscript.

\section{Author details}

${ }^{1}$ School of Life Sciences, Lanzhou University, Lanzhou 730000, China. ${ }^{2}$ Gansu Key Laboratory of Biomonitoring and Bioremediation for Environmental Pollution, Lanzhou 730000, China. ${ }^{3}$ School of Basic Medical Sciences, Lanzhou University, Lanzhou 730000, China. ${ }^{4}$ State Key Laboratory of Genetic Resources and Evolution, Kunming Institute of Zoology, Chinese Academy of Sciences, Kunming 650233, China. ${ }^{5}$ National Nature Reserve of Yanchiwan, Subei 736300, Gansu, China.

\section{Acknowledgements}

We are indebted to all people who helped with the fieldwork at the Yanchiwan National Nature Reserve and to Lanzhou University for permission to carry out field work. Thanks are also given to Dr. Bo Du for statistical assistance, to Dr. Jim Harris for his invaluable suggestions and revision on the original draft of this manuscript and two anonymous referees for their constructive comments. This research was financially supported by a grant from the State Key Laboratory of Genetic Resources and Evolution, Kunming Institute of Zoology, Chinese Academy of Sciences (GREKF13-12). This work was further supported by Yanchiwan National Nature Reserve.

\section{Competing interests}

The authors declare that they have no competing interests.

\section{Ethics statements}

All data collected as part of this study were approved by the Lanzhou University Institutional Animal Care and Use Committee (Approval Numbers:
SCXK-GAN-2013-0003). Field work was approved by authority of the Forestry Department of Gansu Province (Approval Numbers: 201009).

Received: 23 November 2016 Accepted: 26 June 2017

Published online: 20 July 2017

\section{References}

Bentzen RL, Powell AN, Phillips LM, Suydam RS. Incubation behavior of king eiders on the coastal plain of northern Alaska. Polar Biol. 2010;33:1075-82

Bishop MA, Tsamchu D, Li FS. Number and distribution of black-necked cranes wintering in Zhigatse Prefecture. Tibet Chin Birds. 2012;3:191-8.

Bulla M, Valcu M, Rutten AL, Kempenaers B. Biparental incubation patterns in a High-Arctic breeding shorebird: How do pairs divide their duties? Behav Ecol. 2014;25:152-64.

Camfield AF, Martin K. The influence of ambient temperature on horned lark incubation behavior in an alpine environment. Behavior. 2009:146:1615-33.

Conway CJ, Martin TE. Evolution of passerine incubation behavior: influence of food, temperature, and nest predation. Evolution. 2000:54:670-85.

Deeming DC. Patterns and significance of egg turning. In: Deeming DC, editor. Avian incubation behavior, environment, and evolution. Oxford: Oxford University Press; 2002. p. 161-78.

Elibol O, Brake J. Effect of egg turning angle and frequency during incubation on hatchability and incidence of unhatched broiler embryos with head in the small end of the egg. Poult Sci. 2006;85:1433-7.

Kuang FL, Liu N, Zhuoma C, Li JC, Yang L, Li FX. Diurnal time-activity budgets of Grus nigricollis for the pre-laying phase in northern Tibet. J Zhejiang For Coll. 2007;24:686-91 (in Chinese)

Li F, Bishop MA. Ecology and conservation of black-necked cranes (Grus nigricollis). In: Adams NJ, Slotow RH, editors. Proceedings of the XXII international ornithological congress, Durban, South Africa. Johannesburg: Birdlife South Africa; 1999. p. 2533-43.

Magrath MJL, Overveld V, Komdeur J. Contrasting effects of reduced incubation cost on clutch attendance by male and female European starlings. Behavior. 2005;142:1479-93.

Shaffer SA, Clatterbuck CA, Kelsey EC, Naiman AD, Young LC, Vanderwerf EA, Warzybok P, Bradley R, Janhncke J, Bower G. As the egg turns: monitoring egg attendance behavior in wild birds using novel data logging technology. PLoS ONE. 2014;9:e97898.

Smith PA, Tulp I, Schekkerman H, Gilchrist HG, Forbes MR. Shorebird incubation behavior and its influence on the risk of nest predation. Anim Behav. 2012;84:835-42.

Spencer KA, Heidinger BJ, D'Alba LB, Evans NP, Monaghan P. Then versus now: effect of developmental and current environmental conditions on incubation effort in birds. Behav Ecol. 2010;21:999-1004.

Spiegel CS, Haig SM, Goldstein MI, Huso M. Factors affecting incubation patterns and sex roles of black oystercatchers in Alaska. The Condor. 2012;114:123-34.

Weathers WW, Zaun BJ. Egg-turning behavior and nest attentiveness of the endangered Hawaiian goose on Kauai. West Birds. 2010;41:2-9.

Yang R, Wu H, Yang X, Jiang W, Zuo L, Xiang Z. Diurnal time budget of the black-necked crane during the breeding season. Waterbirds. 2007;30:80-5.

Zhang L, Shu M, An B, Zhao C, Suo Y, Yang X. Biparental incubation pattern of the black-necked crane on an alpine plateau. J Ornithol. 2017a;158:697-705.

Zhang L, An B, Shu M, Yang X. Nest-site selection, reproductive ecology and shifts within core-use areas of black-necked cranes at the northern limit of the Tibetan Plateau. PeerJ. 2017b;5:e2939. 